\title{
Theta Functions, Elliptic Hypergeometric Series, and Kawanaka's Macdonald Polynomial Conjecture ${ }^{\star}$
}

\author{
Robin LANGER ${ }^{\dagger}$, Michael J. SCHLOSSER ${ }^{\ddagger}$ and S. Ole WARNAAR ${ }^{\S}$ \\ $\dagger$ Department of Mathematics and Statistics, The University of Melbourne, VIC 3010, Australia \\ E-mail: langer.robin@gmail.com \\ ¥ Fakultät für Mathematik, Universität Wien, Nordbergstrasse 15, A-1090 Vienna, Austria \\ E-mail: michael.schlosser@univie.ac.at \\ $\S$ School of Mathematics and Physics, The University of Queensland, \\ Brisbane, QLD 4072, Australia \\ E-mail: o.warnaar@maths.uq.edu.au
}

Received March 01, 2009, in final form May 19, 2009; Published online May 25, 2009

doi:10.3842/SIGMA.2009.055

\begin{abstract}
We give a new theta-function identity, a special case of which is utilised to prove Kawanaka's Macdonald polynomial conjecture. The theta-function identity further yields a transformation formula for multivariable elliptic hypergeometric series which appears to be new even in the one-variable, basic case.
\end{abstract}

Key words: theta functions; Macdonald polynomials; elliptic hypergeometric series

2000 Mathematics Subject Classification: 05E05; 33D52; 33D67

\section{Introduction}

The recent discovery of elliptic hypergeometric series (EHS) by Frenkel and Turaev [2] has led to a renewed interest in theta-function identities. Such identities are at the core of many of the proofs of identities for EHS associated with root systems. If, for $|p|<1$ and $x \in \mathbb{C}^{*}$,

$$
\theta(x)=\theta(x ; p)=\prod_{k=0}^{\infty}\left(1-x p^{k}\right)\left(1-x^{-1} p^{k+1}\right)
$$

is a normalised theta function, then three examples of theta function identities featured in the theory of EHS are

$$
\begin{aligned}
& \sum_{i=1}^{n} \frac{\prod_{j=1}^{n} \theta\left(x_{i} / y_{j}\right)}{\prod_{j=1, j \neq i}^{n} \theta\left(x_{i} / x_{j}\right)}=0 \quad \text { for } \quad x_{1} \cdots x_{n}=y_{1} \cdots y_{n}, \\
& \sum_{i=1}^{n} \frac{x_{i} \prod_{j=1}^{n-2} \theta\left(x_{i} / y_{j}\right) \theta\left(x_{i} y_{j}\right)}{\prod_{j=1, j \neq i}^{n} \theta\left(x_{i} / x_{j}\right) \theta\left(x_{i} x_{j}\right)}=0 \quad \text { for } n \geq 2,
\end{aligned}
$$

* This paper is a contribution to the Proceedings of the Workshop "Elliptic Integrable Systems, Isomonodromy Problems, and Hypergeometric Functions" (July 21-25, 2008, MPIM, Bonn, Germany). The full collection is available at http://www.emis.de/journals/SIGMA/Elliptic-Integrable-Systems.html 
and

$$
\sum_{\substack{I \subseteq[n] \\|\bar{I}|=r}} \prod_{\substack{i \in I \\ j \in[n]}} \frac{\theta\left(x_{i} y_{j}\right)}{\theta\left(q x_{i} y_{j}\right)} \prod_{\substack{i \in I \\ j \notin I}} \frac{\theta\left(q x_{i} / x_{j}\right)}{\theta\left(x_{i} / x_{j}\right)}=\sum_{\substack{I \subseteq[n] \\|\bar{I}|=r}} \prod_{\substack{i \in I \\ j \in[n]}} \frac{\theta\left(y_{i} x_{j}\right)}{\theta\left(q y_{i} x_{j}\right)} \prod_{\substack{i \in I \\ j \notin I}} \frac{\theta\left(q y_{i} / y_{j}\right)}{\theta\left(y_{i} / y_{j}\right)}
$$

where $[n]:=\{1,2, \ldots, n\}$. The identity (1.1) is standard and can for example be found in the classic text of Whittaker and Watson [24, page 451]. It was employed by Gustafson in [4] to derive an $\mathrm{A}_{n-1}$ extension of Bailey's very-well-poised ${ }_{6} \psi_{6}$ summation. In the same paper, Gustafson discovered the identity (1.2) [4, Lemma 4.14] and used it to derive a $\mathrm{C}_{n}$ extension of Bailey's very-well-poised ${ }_{6} \psi_{6}$ summation. The identities (1.1) and (1.2) were also employed by Rosengren in [14] to prove elliptic analogues of Milne's $\mathrm{A}_{n-1}$ Jackson sum [12] and Schlosser's $\mathrm{D}_{n}$ Jackson sum [20]. The identity (1.3) was only recently discovered by Kajihara and Noumi [6, Theorem 1.3] by combining symmetries of the Frobenius determinant with operator methods. They used it to extend Rosengren's elliptic $A_{n-1}$ Jackson summation to a transformation between EHS on A-type root systems of different rank, see [6, Theorem 2.1]. Ruijsenaars, in his study of integrable systems of Calogero-Moser type, also discovered the identity (1.3), see [18, Equation (2.44)], where it appeared in the form of a functional equation encoding the commutativity of analytic difference operators of type $\mathrm{A}_{n-1}$. Earlier, he had used a special case of this identity (Equation (2.4) in [17], see also [19]) to show the commutativity of what is nowadays known as the Macdonald-Ruijsenaars difference operator [17].

Let

$$
(a)_{n}=(a ; q, p)_{n}=\prod_{k=0}^{n-1} \theta\left(a q^{k} ; p\right)
$$

be a theta shifted factorial (cf. [3, Chapter 11]), and set

$$
\left(a_{1}, \ldots, a_{k}\right)_{n}=\left(a_{1}\right)_{n} \cdots\left(a_{k}\right)_{n} .
$$

Then the main result of this paper is the following new identity for theta functions.

Theorem 1.1. For $n$ a nonnegative integer and $v, w, q, t, x_{1}, \ldots, x_{n} \in \mathbb{C}^{*}$ such that both sides are well defined,

$$
\begin{aligned}
& \sum_{r=0}^{n} \frac{(v, w)_{r}}{(q v / t, q w / t)_{r}} \sum_{\substack{I \subseteq[n] \\
|I|=r}} \prod_{i \in I} \frac{q \theta\left(v x_{i} / t w\right) \theta\left(t q^{-r} x_{i} / q w\right)}{t \theta\left(v x_{i} / q w\right) \theta\left(q^{-r} x_{i} / w\right)} \\
& \quad \times \prod_{j \notin I} \frac{\theta\left(x_{j} / q\right) \theta\left(q^{-r} x_{j} / t w\right)}{\theta\left(x_{j} / t\right) \theta\left(q^{-r} x_{j} / q w\right)} \prod_{\substack{i \in I \\
j \notin I}} \frac{\theta\left(t x_{i} / q x_{j}\right) \theta\left(q x_{i} / x_{j}\right)}{\theta\left(x_{i} / x_{j}\right) \theta\left(t x_{i} / x_{j}\right)} \\
& =\sum_{r=0}^{n} \frac{(v, w)_{r}}{(q v / t, q w / t)_{r}} \sum_{\substack{I \subseteq[n] \\
|I|=r}} \prod_{i \in I} \frac{q \theta\left(x_{i} / q\right) \theta\left(q^{r} v x_{i} / t^{2}\right)}{t \theta\left(x_{i} / t\right) \theta\left(q^{r} v x_{i} / t q\right)} \\
& \quad \times \prod_{j \notin I} \frac{\theta\left(v x_{j} / t w\right) \theta\left(q^{r} v x_{j} / q\right)}{\theta\left(v x_{j} / q w\right) \theta\left(q^{r} v x_{j} / t\right)} \prod_{\substack{i \in I \\
j \notin I}} \frac{\theta\left(t x_{j} / q x_{i}\right) \theta\left(q x_{j} / x_{i}\right)}{\theta\left(x_{j} / x_{i}\right) \theta\left(t x_{j} / x_{i}\right)} .
\end{aligned}
$$

After clearing denominators and replacing $\left(t, v, w, x_{1}\right) \mapsto\left(t q, t v, w^{-1}, q t x\right)$ the $n=1$ case of the theorem takes the form

$$
\theta(v) \theta(t x) \theta(t w) \theta(w x) \theta(t v x) \theta\left(t^{2} v w x\right)+\theta(w) \theta(x) \theta(t v) \theta(t v x) \theta\left(t^{2} w x\right) \theta(t v w x)
$$




$$
=\theta(v) \theta(x) \theta(t w) \theta(t w x) \theta\left(t^{2} v x\right) \theta(t v w x)+\theta(w) \theta(t x) \theta(t v) \theta(v x) \theta(t w x) \theta\left(t^{2} v w x\right) .
$$

This formula, which also follows from the $(n, r)=(2,1)$ case of (1.3) upon clearing denominators and substituting $\left(x_{2}, y_{1}, y_{2}, q\right) \mapsto\left(t w x x_{1}, v / x_{1}, 1 / t x x_{1}, t\right)$, is a once-iterated version of the Riemann relation ${ }^{1}$

$$
\theta(x z) \theta(x / z) \theta(y w) \theta(y / w)-\theta(x w) \theta(x / w) \theta(y z) \theta(y / z)=\frac{y}{z} \theta(x y) \theta(x / y) \theta(z w) \theta(z / w) .
$$

We remark that formula (1.4) is a four-term identity involving four free parameters, each term containing a product of six theta functions. For comparison, the formula (1.1) ((1.2)) for $n=4$ is a four-term identity involving seven (six) free parameters, each term containing a product of seven (ten) theta functions.

As will be shown in Section 4, Theorem 1.1 can be used to obtain identities for EHS for the root systems of type A. More unexpectedly, however, it also implies a combinatorial identity in the theory of Macdonald polynomials [11, Chapter VI], conjectured in 1999 by Kawanaka [8]. For $\lambda$ a partition let $P_{\lambda}(x ; q, t)$ be the Macdonald symmetric function in countably many independent variables $x=\left(x_{1}, x_{2}, \ldots\right)$. Let $a(s)$ and $l(s)$ be the arm-length and leg-length of the square $s$ in the diagram of $\lambda$, and let

$$
(a ; q)_{\infty}=\prod_{k=0}^{\infty}\left(1-a q^{k}\right)
$$

be a $q$-shifted factorial.

Conjecture 1.2 (Kawanaka). The following formal identity holds:

$$
\sum_{\lambda} \prod_{s \in \lambda}\left(\frac{1+q^{a(s)} t^{l(s)+1}}{1-q^{a(s)+1} t^{l(s)}}\right) P_{\lambda}\left(x ; q^{2}, t^{2}\right)=\prod_{i \geq 1} \frac{\left(-t x_{i} ; q\right)_{\infty}}{\left(x_{i} ; q\right)_{\infty}} \prod_{i<j} \frac{\left(t^{2} x_{i} x_{j} ; q^{2}\right)_{\infty}}{\left(x_{i} x_{j} ; q^{2}\right)_{\infty}} .
$$

Using elementary results from Macdonald polynomial theory and (a special limiting case of) Theorem 1.1 we can claim the following.

Theorem 1.3. Kawanaka's conjecture is true.

We finally remark that although Theorem 1.1 is new, a special limiting case coincides with a limiting case of another theta-function identity, implicit in [14, Corollary 5.3].

Theorem 1.4 (Rosengren). For $n$ a nonnegative integer and $v, w, y, z, q, x_{1}, \ldots, x_{n} \in \mathbb{C}^{*}$ such that $v w=q^{n-1} y z$ and such that both sides are well defined,

$$
\begin{aligned}
& \sum_{r=0}^{n}(-1)^{r} q^{\left(\begin{array}{c}
r+1 \\
2
\end{array}\right)-n r} \frac{(v, w)_{r}}{(y, z)_{r}} \sum_{\substack{I \subseteq[n] \\
|I|=r}} \prod_{i \in I} \frac{\theta\left(y x_{i}\right) \theta\left(z x_{i}\right)}{\theta\left(q^{1-r} x_{i}\right)} \prod_{j \notin I} \frac{\theta\left(v x_{j}\right) \theta\left(w x_{j}\right)}{\theta\left(q^{-r} x_{j}\right)} \prod_{\substack{i \in I \\
j \notin I}} \frac{\theta\left(q x_{i} / x_{j}\right)}{\theta\left(x_{i} / x_{j}\right)} \\
& \quad=\frac{(y / v, y / w)_{n}}{(y, y / v w)_{n}} \prod_{i=1}^{n} \theta\left(v w x_{i}\right) .
\end{aligned}
$$

If we set $p=0$ in Theorems 1.1 and Theorem 1.4 using $\theta(a ; 0)=(1-a)$, and then let $t \rightarrow \infty$ in the former (so that its right-hand side vanishes unless $r=0$ ) and $(y, z) \rightarrow(0, \infty)$ (such that $y z=q^{1-n} v w$ ) in the latter, we obtain one and the same rational function identity (up to a rescaling of $x_{i} \mapsto w q x_{i}$ in Theorem 1.1).

\footnotetext{
${ }^{1}$ Riemann gave a number of addition formulae for theta functions on arbitrary genus Riemann surfaces, but it is not clear he actually was the first to discover (1.5).
} 


\section{Proof of Theorem 1.1}

\subsection{Preliminary remarks}

Let the left- and right-hand sides of the identity of the theorem be denoted as $L(x ; v, w, q, t ; p)$ and $R(x ; v, w, q, t ; p)$ respectively, where $x:=\left(x_{1}, \ldots, x_{n}\right)$. Then, by

$$
\theta(z)=-z \theta\left(z^{-1}\right)
$$

and

$$
\frac{\left(a ; q^{-1}, p\right)_{k}}{\left(b ; q^{-1}, p\right)_{k}}=\frac{\left(a^{-1} ; q, p\right)_{k}}{\left(b^{-1} ; q, p\right)_{k}}\left(\frac{a}{b}\right)^{k},
$$

it follows that

$$
L(x ; v, w, q, t ; p)=R\left(q^{-1} t^{-1} v w^{-1} x ; v^{-1}, w^{-1}, q^{-1}, t^{-1} ; p\right) .
$$

Hence Theorem 1.1 may be reformulated as the symmetry

$$
L(x ; v, w, q, t ; p)=L\left(q^{-1} t^{-1} v w^{-1} x ; v^{-1}, w^{-1}, q^{-1}, t^{-1} ; p\right) .
$$

Alternatively, by

$$
\frac{(a)_{n-k}}{(b)_{n-k}}=\frac{(a)_{n}\left(q^{1-n} / b\right)_{k}}{(b)_{n}\left(q^{1-n} / a\right)_{k}}\left(\frac{b}{a}\right)^{k},
$$

and the substitutions $I \mapsto[n]-I$ and $r \mapsto n-r$, it follows that

$$
L(x ; v, w, q, t ; p)=R\left(x ; q^{-n} t w^{-1}, q^{-n} t v^{-1}, q, t ; p\right) \frac{(v, w)_{n}}{(q v / t, q w / t)_{n}}\left(\frac{q}{t}\right)^{n} .
$$

Hence Theorem 1.1 is also equivalent to

$$
L(x ; v, w, q, t ; p)=L\left(x ; q^{-n} t w^{-1}, q^{-n} t v^{-1}, q, t ; p\right) \frac{(v, w)_{n}}{(q v / t, q w / t)_{n}}\left(\frac{q}{t}\right)^{n} .
$$

\subsection{Proof of Theorem 1.1}

Recall that $x=\left(x_{1}, \ldots, x_{n}\right)$. We begin by introducing a scalar variable $u$ in the theorem by making the substitution $x \mapsto x / u$. Then

$$
\begin{aligned}
\sum_{r=0}^{n} \frac{(v, w)_{r}}{(q v / t, q w / t)_{r}} \sum_{\substack{I \subseteq[n] \\
|I|=r}} \prod_{\substack{i \in I \\
\text { I }}} \frac{q \theta\left(v x_{i} / t u w\right) \theta\left(t q^{-r} x_{i} / q u w\right)}{t \theta\left(v x_{i} / q u w\right) \theta\left(q^{-r} x_{i} / u w\right)} \\
\quad \times \prod_{j \notin I} \frac{\theta\left(x_{j} / q u\right) \theta\left(q^{-r} x_{j} / t u w\right)}{\theta\left(x_{j} / t u\right) \theta\left(q^{-r} x_{j} / q u w\right)} \prod_{\substack{i \in I \\
j \notin I}} \frac{\theta\left(t x_{i} / q x_{j}\right) \theta\left(q x_{i} / x_{j}\right)}{\theta\left(x_{i} / x_{j}\right) \theta\left(t x_{i} / x_{j}\right)} \\
=\sum_{r=0}^{n} \frac{(v, w)_{r}}{(q v / t, q w / t)_{r}} \sum_{\substack{I \subseteq[n] \\
|I|=r}} \prod_{i \in I} \frac{q \theta\left(x_{i} / q u\right) \theta\left(q^{r} v x_{i} / t^{2} u\right)}{t \theta\left(x_{i} / t u\right) \theta\left(q^{r} v x_{i} / t q u\right)} \\
\quad \times \prod_{j \notin I} \frac{\theta\left(v x_{j} / t u w\right) \theta\left(q^{r} v x_{j} / q u\right)}{\theta\left(v x_{j} / q u w\right) \theta\left(q^{r} v x_{j} / t u\right)} \prod_{\substack{i \in I \\
j \notin I}} \frac{\theta\left(t x_{j} / q x_{i}\right) \theta\left(q x_{j} / x_{i}\right)}{\theta\left(x_{j} / x_{i}\right) \theta\left(t x_{j} / x_{i}\right)} .
\end{aligned}
$$


Let $\mathcal{L}(x ; u, v, w, q, t ; p)$ and $\mathcal{R}(x ; u, v, w, q, t ; p)$ denote the left-hand side and right-hand side of this identity, and further define

$$
\mathcal{F}=\mathcal{L}-\mathcal{R}
$$

Comparing with our earlier definitions we thus have

$$
\mathcal{L}(x ; u, v, w, q, t ; p)=L(x / u ; v, w, q, t ; p) \quad \text { and } \quad \mathcal{R}(x ; u, v, w, q, t ; p)=R(x / u ; v, w, q, t ; p) .
$$

We are mainly interested in the $u$-dependence of $\mathcal{L}, \mathcal{R}$ and $\mathcal{F}$, and will frequently write $\mathcal{L}(u)$, $\mathcal{R}(u)$ and $\mathcal{F}(u)$. The claim of the theorem is thus $\mathcal{F}(u)=0$, which will be proved by induction on $n$, the cardinality of the alphabet $x$.

For $n=0$ the theorem is trivial: $\mathcal{F}(-; u, v, w, q, t ; p)=1-1=0$.

From $\theta(z)=-z \theta(p z)$ it immediately follows that $\mathcal{F}$ is periodic along annuli, with period $p$ :

$$
\mathcal{F}(p u)=\mathcal{F}(u) .
$$

The function $\mathcal{F}(u)$ has simple poles at

$$
u= \begin{cases}x_{i} p^{k} / t, & \\ v x_{i} p^{k} / q w, & \\ q^{-m} p^{k} x_{i} / w & \text { for } m \in[r], \\ q^{m-1} p^{k} v x_{i} / t & \text { for } m \in[r],\end{cases}
$$

where $i \in[n]$ and $k \in \mathbb{Z}$. If we show that these poles all have zero residue then, by (2.3) and Liouville's theorem, $\mathcal{F}(u)$ must be constant. By the periodicity (2.3) it suffices to consider the poles (2.4) with $k=0$. By the symmetry of $\mathcal{F}(x ; u, v, w, q, t ; p)$ in $x$ it also suffices to only consider $i=n$. From (2.2) it follows that

$$
\mathcal{L}(x ; u, v, w, q, t ; p)=\mathcal{R}\left(x ; q t u v^{-1} w, v^{-1}, w^{-1}, q^{-1}, t^{-1} ; p\right) .
$$

It is thus sufficient to only consider the residues of the poles at

$$
u=\left\{\begin{array}{l}
x_{n} / t, \\
q^{-m} x_{n} / w \text { for } m \in[r] .
\end{array}\right.
$$

First we compute the residue at $u=x_{n} / t$. For $\mathcal{L}(u)$ only terms such that $n \notin I$ contribute and for $\mathcal{R}(u)$ only terms with $n \in I$ do. Hence, after an elementary calculation which includes the use of (2.1),

$$
\lim _{u \rightarrow a} \frac{u-a}{\theta(a / u ; p)}=\frac{a}{(p ; p)_{\infty}^{2}}
$$

and a shift $r \mapsto r+1$ in $\mathcal{R}(u)$, we find

$$
\begin{aligned}
& \operatorname{Res}_{u=t^{-1} x_{n}} \mathcal{F}(x ; u, v, w, q, t ; p) \\
& \quad=\mathcal{F}\left(x^{(n)} ; x_{n} / q t, v, q w, q, t ; p\right) \frac{q x_{n}}{t^{2}} \frac{1}{(p ; p)_{\infty}^{2}} \frac{\theta(w) \theta(t / q)}{\theta(q w / t)} \prod_{i=1}^{n-1} \frac{\theta\left(q x_{i} / x_{n}\right) \theta\left(t x_{i} / q x_{n}\right)}{\theta\left(t x_{i} / x_{n}\right) \theta\left(x_{i} / x_{n}\right)}
\end{aligned}
$$

where $x^{(n)}:=\left(x_{1}, \ldots, x_{n-1}\right)$. By induction on $n$ this vanishes. 
Next we consider the pole at $u=q^{-m} x_{n} / w$. The only contributions to its residue come from $\mathcal{L}(u)$ with (i) $n \in I$ and $r=m$ or (ii) $n \notin I$ and $r=m-1$. An elementary calculation shows that these two contributions are the same up to a sign, and thus cancel.

Now that we have established that all poles of $\mathcal{F}(u)$ have zero residue we may conclude that $\mathcal{F}(u)$ is independent of $u$. To show that it is actually identically zero we take $u=x_{n} / q$. In $\mathcal{L}\left(x_{n} / q\right)$ only terms such that $n \in I$ contribute and in $\mathcal{R}\left(x_{n} / q\right)$ only terms with $n \notin I$ do. Again using (2.1) and making a shift $r \mapsto r+1$ in $\mathcal{L}\left(x_{n} / q\right)$, we find

$$
\mathcal{F}\left(x ; x_{n} / q, v, w, q, t ; p\right)=\mathcal{F}\left(x^{(n)} ; x_{n}, q v, w, q, t ; p\right) \frac{\theta(v) \theta(q v / t w)}{\theta(q v / t) \theta(v / w)} .
$$

By induction this once again vanishes.

\section{Proof of Kawanaka's conjecture}

\subsection{Preliminary remarks}

Kawanaka's identity complements a set of four Macdonald polynomial identities discovered by Macdonald [11, page 349]. In slightly more general form as given in [23], these identities may be stated as the following pair of results (Macdonald's formulae correspond to $b=0$ and $b=1$ )

$$
\begin{aligned}
& \sum_{\lambda} b^{c(\lambda)} \prod_{\substack{s \in \lambda \\
l(s) \text { even }}}\left(\frac{1-q^{a(s)} t^{l(s)+1}}{1-q^{a(s)+1} t^{l(s)}}\right) P_{\lambda}(x ; q, t)=\prod_{i \geq 1} \frac{\left(b t x_{i} ; q\right)_{\infty}}{\left(b x_{i} ; q\right)_{\infty}} \prod_{i<j} \frac{\left(t x_{i} x_{j} ; q\right)_{\infty}}{\left(x_{i} x_{j} ; q\right)_{\infty}}, \\
& \sum_{\lambda} b^{r(\lambda)} \prod_{\substack{s \in \lambda \\
a(s) \text { odd }}}\left(\frac{1-q^{a(s)} t^{l(s)+1}}{1-q^{a(s)+1} t^{l(s)}}\right) P_{\lambda}(x ; q, t)=\prod_{i \geq 1} \frac{\left(1+b x_{i}\right)\left(q t x_{i}^{2} ; q^{2}\right)_{\infty}}{\left(x_{i}^{2} ; q^{2}\right)_{\infty}} \prod_{i<j} \frac{\left(t x_{i} x_{j} ; q\right)_{\infty}}{\left(x_{i} x_{j} ; q\right)_{\infty}},
\end{aligned}
$$

where $c(\lambda)$ and $r(\lambda)$ are the number of columns and rows of odd length, respectively. Due to its quadratic nature Kawanaka's identity is significantly harder to prove than (3.1).

If $x$ contains a single variable then (1.6) simplifies to the classical $q$-binomial theorem [3, Equation (II.3)]

$$
\sum_{k=0}^{\infty} \frac{(-t ; q)_{k}}{(q ; q)_{k}} x^{k}=\frac{(-t x ; q)_{\infty}}{(x ; q)_{\infty}}
$$

where, for integer $k$,

$$
(a ; q)_{k}=\frac{(a ; q)_{\infty}}{\left(a q^{k} ; q\right)_{\infty}} .
$$

If $q=t$ then (1.6) reduces to an identity for the Schur function $s_{\lambda}$ proved by Kawanaka [8]. Specifically, using that $P_{\lambda}(x ; q, q)=s_{\lambda}(x)$ and $a(s)+l(s)+1=h(s)$ with $h(s)$ the hook-length of the square $s$, it follows that the $q=t$ case of (1.6) is

$$
\sum_{\lambda} \prod_{s \in \lambda}\left(\frac{1+q^{h(s)}}{1-q^{h(s)}}\right) s_{\lambda}(x)=\prod_{i \geq 1} \frac{\left(-q x_{i} ; q\right)_{\infty}}{\left(x_{i} ; q\right)_{\infty}} \prod_{i<j} \frac{1}{1-x_{i} x_{j}} .
$$

This result was reproved and reinterpreted by Rosengren in [15]. If $Q_{\mu}$ is Schur's $Q$-function, see [11, Section III.8], then Rosengren observed that for $\mu$ a partition of length $m$

$$
Q_{\mu}\left(1, q, q^{2}, \ldots\right)=\left(\frac{(-1 ; q)_{\infty}}{(q ; q)_{\infty}}\right)^{m} \prod_{1 \leq i<j \leq m}\left(q^{\mu_{i}}-q^{\mu_{j}}\right) \sum_{\lambda} \prod_{s \in \lambda}\left(\frac{1+q^{h(s)}}{1-q^{h(s)}}\right) s_{\lambda}\left(-q^{\mu_{1}}, \ldots,-q^{\mu_{m}}\right) .
$$


By (3.3) this results in a product-form for $Q_{\mu}\left(1, q, q^{2}, \ldots\right)$ and, consequently, in a product-form for the generating function of marked shifted tableaux [15, Corollary 3.1]. It is an open problem to find a corresponding interpretation of (1.6).

Another special case of (1.6) proved by Kawanaka corresponds to $q=0$ [7]. Using $P_{\lambda}(x ; 0, t)=$ $P_{\lambda}(x ; t)$ with on the right a Hall-Littlewood symmetric function, it follows that the $q=0$ case of $(1.6)$ is

$$
\sum_{\lambda}\left(\prod_{i \geq 1}\left(-t ; t^{2}\right)_{m_{i}(\lambda)}\right) P_{\lambda}\left(x ; t^{2}\right)=\prod_{i \geq 1} \frac{1+t x_{i}}{1-x_{i}} \prod_{i<j} \frac{1-t^{2} x_{i} x_{j}}{1-x_{i} x_{j}},
$$

with $m_{i}(\lambda)$ the multiplicity of the part $i$ in $\lambda$. This is in fact a special case of a much more general identity for Hall-Littlewood functions proved in [23, Theorem 1.1]. So far our attempts to generalise (1.6) to include this more general result for Hall-Littlewood functions have been unsuccessful.

\subsection{Macdonald polynomials}

Let $\lambda=\left(\lambda_{1}, \lambda_{2}, \ldots\right)$ be a partition, i.e., $\lambda_{1} \geq \lambda_{2} \geq \cdots$ with finitely many $\lambda_{i}$ unequal to zero. The length and weight of $\lambda$, denoted by $l(\lambda)$ and $|\lambda|$, are the number and sum of the non-zero $\lambda_{i}$ respectively. As usual we identify two partitions that differ only in their string of zeros, so that $(6,3,3,1,0,0)$ and $(6,3,3,1)$ represent the same partition. When $|\lambda|=N$ we say that $\lambda$ is a partition of $N$, and the unique partition of zero is denoted by 0 . The multiplicity of the part $i$ in the partition $\lambda$ is denoted by $m_{i}(\lambda)$.

We identify a partition with its Ferrers graph, defined by the set of points in $(i, j) \in \mathbb{Z}^{2}$ such that $1 \leq j \leq \lambda_{i}$, and further make the usual identification between Ferrers graphs and (Young) diagrams by replacing points by squares. The conjugate $\lambda^{\prime}$ of $\lambda$ is the partition obtained by reflecting the diagram of $\lambda$ in the main diagonal.

The dominance partial order on the set of partitions of $N$ is defined by $\lambda \geq \mu$ if $\lambda_{1}+\cdots+\lambda_{i} \geq$ $\mu_{1}+\cdots+\mu_{i}$ for all $i \geq 1$. If $\lambda \geq \mu$ and $\lambda \neq \mu$ then $\lambda>\mu$.

If $\lambda$ and $\mu$ are partitions then $\mu \subseteq \lambda$ if (the diagram of) $\mu$ is contained in (the diagram of) $\lambda$, i.e., $\mu_{i} \leq \lambda_{i}$ for all $i \geq 1$. If $\mu \subseteq \lambda$ then the skew-diagram $\lambda-\mu$ denotes the set-theoretic difference between $\lambda$ and $\mu$, i.e., those squares of $\lambda$ not contained in $\mu$. The skew diagram $\lambda-\mu$ is a vertical $r$-strip if $|\lambda-\mu|:=|\lambda|-|\mu|=r$ and if, for all $i \geq 1, \lambda_{i}-\mu_{i}$ is at most one, i.e., each row of $\lambda-\mu$ contains at most one square. For example, if $\lambda=(5,4,2,2,1)$ and $\mu=(4,3,1,1,1)$ then $\lambda-\mu$ is a vertical 4 -strip. The set of all vertical $r$-strips is denoted by $\mathcal{V}_{r}$ and the set of all vertical strips by $\mathcal{V}=\bigcup_{r=0}^{\infty} \mathcal{V}_{r}$. The skew diagram $\lambda-\mu$ is a horizontal $r$-strip if $|\lambda-\mu|=r$ and if, for all $i \geq 1, \lambda_{i}^{\prime}-\mu_{i}^{\prime}$ is at most one, i.e., each column of $\lambda-\mu$ contains at most one square. The set of all horizontal $r$-strips is denoted by $\mathcal{H}_{r}$ and the set of all horizontal strips by $\mathcal{H}$.

Let $s=(i, j)$ be a square in the diagram of $\lambda$, and let $a(s)$ and $l(s)$ be the arm-length and leg-length of $s$, given by

$$
a(s)=\lambda_{i}-j, \quad l(s)=\lambda_{j}^{\prime}-i .
$$

Then we define the rational functions $b_{\lambda}^{+}(q, t)$ and $b_{\lambda}^{-}(q, t)$ as

$$
b_{\lambda}^{ \pm}(q, t)=\prod_{s \in \lambda} \frac{1 \mp q^{a(s)} t^{l(s)+1}}{1-q^{a(s)+1} t^{l(s)}} .
$$

The function $b_{\lambda}^{+}(q, t)$ is standard in Macdonald polynomial theory and is usually denoted as $b_{\lambda}(q, t)$. Below we use both notations: $b_{\lambda}=b_{\lambda}^{+}$. Note that $b_{\lambda}^{-}(q, t)$ corresponds to the product in 
the summand of Kawanaka's conjecture. Since under conjugation arms and legs are interchanged, it easily follows that

$$
b_{\lambda^{\prime}}^{-}(q, t)=b_{\lambda}^{-}(t, q) / b_{\lambda}\left(t^{2}, q^{2}\right) .
$$

Subsequently we require non-combinatorial expressions for both $b_{\lambda}^{-}$and $b_{\lambda^{\prime}}^{-}$. From (3.4) it follows that

$$
b_{\lambda}^{ \pm}(q, t)=\prod_{i=1}^{n} \frac{\left( \pm t^{n-i+1} ; q\right)_{\lambda_{i}}}{\left(q t^{n-i} ; q\right)_{\lambda_{i}}} \prod_{1 \leq i<j \leq n} \frac{\left( \pm t^{j-i}, q t^{j-i} ; q\right)_{\lambda_{i}-\lambda_{j}}}{\left( \pm t^{j-i+1}, q t^{j-i-1} ; q\right)_{\lambda_{i}-\lambda_{j}}}
$$

where $n$ is an integer such that $l(\lambda) \leq n$. From this and (3.5) we also find

$$
b_{\lambda^{\prime}}^{-}(q, t)=\prod_{i=1}^{n} \frac{\left(-t q^{n-i} ; t\right)_{\lambda_{i}}}{\left(q^{n-i+1} ; t\right)_{\lambda_{i}}} \prod_{1 \leq i<j \leq n} \frac{\left(q^{j-i+1},-t q^{j-i-1} ; t\right)_{\lambda_{i}-\lambda_{j}}}{\left(q^{j-i},-t q^{j-i} ; t\right)_{\lambda_{i}-\lambda_{j}}}
$$

where, again, $l(\lambda) \leq n$.

Let $\mathfrak{S}_{n}$ denote the symmetric group, acting on $x=\left(x_{1}, \ldots, x_{n}\right)$ by permuting the $x_{i}$, and let $\Lambda_{n}=\mathbb{Z}\left[x_{1}, \ldots, x_{n}\right]^{\mathfrak{S}_{n}}$ and $\Lambda$ denote the ring of symmetric polynomials in $n$ independent variables and the ring of symmetric functions in countably many variables, respectively.

For $\lambda=\left(\lambda_{1}, \ldots, \lambda_{n}\right)$ a partition of at most $n$ parts the monomial symmetric function $m_{\lambda}$ is defined as

$$
m_{\lambda}(x)=\sum x^{\alpha}
$$

where the sum is over all distinct permutations $\alpha$ of $\lambda$, and $x^{\alpha}=x_{1}^{\alpha_{1}} \cdots x_{n}^{\alpha_{n}}$. For $l(\lambda)>n$ we set $m_{\lambda}(x)=0$. The monomial symmetric functions $m_{\lambda}$ for $l(\lambda) \leq n$ form a $\mathbb{Z}$-basis of $\Lambda_{n}$.

For $r$ a nonnegative integer the power sums $p_{r}$ are given by $p_{0}=1$ and $p_{r}=m_{(r)}$ for $r>1$. Hence

$$
p_{r}(x)=\sum_{i \geq 1} x_{i}^{r}
$$

More generally the power-sum products are defined as $p_{\lambda}(x)=p_{\lambda_{1}}(x) \cdots p_{\lambda_{n}}(x)$.

Define the Macdonald scalar product $\langle\cdot, \cdot\rangle_{q, t}$ on the ring of symmetric functions by

$$
\left\langle p_{\lambda}, p_{\mu}\right\rangle_{q, t}=\delta_{\lambda \mu} z_{\lambda} \prod_{i=1}^{n} \frac{1-q^{\lambda_{i}}}{1-t^{\lambda_{i}}}
$$

with $z_{\lambda}=\prod_{i \geq 1} m_{i} ! i^{m_{i}}$ and $m_{i}=m_{i}(\lambda)$. If we denote the ring of symmetric functions in $n$ variables over the field $\mathbb{F}=\mathbb{Q}(q, t)$ of rational functions in $q$ and $t$ by $\Lambda_{n, \mathbb{F}}$, then the Macdonald polynomial $P_{\lambda}(x ; q, t)$ is the unique symmetric polynomial in $\Lambda_{n, \mathbb{F}}$ such that [11, Section VI.4, Equation (4.7)]:

$$
P_{\lambda}(x ; q, t)=m_{\lambda}(x)+\sum_{\mu<\lambda} u_{\lambda \mu}(q, t) m_{\mu}(x)
$$

and

$$
\left\langle P_{\lambda}, P_{\mu}\right\rangle_{q, t}=0 \quad \text { if } \quad \lambda \neq \mu
$$

The Macdonald polynomials $P_{\lambda}(x ; q, t)$ with $l(\lambda) \leq n$ form an $\mathbb{F}$-basis of $\Lambda_{n, \mathbb{F}}$. If $l(\lambda)>n$ then $P_{\lambda}(x ; q, t)=0$. 
Since $P_{\lambda}\left(x_{1}, \ldots, x_{n}, 0 ; q, t\right)=P_{\lambda}\left(x_{1}, \ldots, x_{n} ; q, t\right)$ one can extend the Macdonald polynomials to symmetric functions containing an infinite number of independent variables $x=\left(x_{1}, x_{2}, \ldots\right)$, to obtain a basis of $\Lambda_{\mathbb{F}}=\Lambda \otimes \mathbb{F}$.

A second Macdonald symmetric function is defined as

$$
Q_{\lambda}(x ; q, t)=b_{\lambda}(q, t) P_{\lambda}(x ; q, t) .
$$

The normalisation of the Macdonald inner product is then

$$
\left\langle P_{\lambda}, Q_{\lambda}\right\rangle_{q, t}=1
$$

Important in the proof of Kawanaka's conjecture are the Pieri rules for Macdonald polynomials. Let $g_{r}(x ; q, t):=Q_{(r)}(x ; q, t)$, or equivalently, [11, Section VI.2, Equation (2.8)]

$$
\prod_{i=1}^{n} \frac{\left(t x_{i} y ; q\right)_{\infty}}{\left(x_{i} y ; q\right)_{\infty}}=\sum_{r=0}^{\infty} g_{r}(x ; q, t) y^{r}
$$

Then the Pieri coefficients $\phi_{\mu / \nu}$ and $\psi_{\mu / \nu}$ are given by [11, Section VI.6, Equation (6.24)]

$$
\begin{aligned}
P_{\nu}(x ; q, t) g_{r}(x ; q, t) & =\sum_{\substack{\mu \in \mathcal{H}_{r} \\
\mu-\nu \in \mathcal{H}_{r}}} \phi_{\mu / \nu}(q, t) P_{\mu}(x ; q, t), \\
Q_{\nu}(x ; q, t) g_{r}(x ; q, t) & =\sum_{\substack{\mu \\
\mu-\nu \in \mathcal{H}_{r}}} \psi_{\mu / \nu}(q, t) Q_{\mu}(x ; q, t) .
\end{aligned}
$$

\subsection{Proof of Theorem 1.3}

By (3.4) Kawanaka's conjecture can be stated as

$$
\sum_{\lambda} b_{\lambda}^{-}(q, t) P_{\lambda}\left(x ; q^{2}, t^{2}\right)=\prod_{i \geq 1} \frac{\left(-t x_{i} ; q\right)_{\infty}}{\left(x_{i} ; q\right)_{\infty}} \prod_{i<j} \frac{\left(t^{2} x_{i} x_{j} ; q^{2}\right)_{\infty}}{\left(x_{i} x_{j} ; q^{2}\right)_{\infty}} .
$$

Our initial steps closely follow Macdonald's proof of (3.1). It suffices to prove (3.10) for the finite set of variables $x=\left(x_{1}, \ldots, x_{n}\right)$. If we also denote $x^{\prime}=\left(x_{1}, \ldots, x_{n}, y\right)$ and let

$$
\Phi(x ; q, t):=\sum_{\lambda} b_{\lambda}^{-}(q, t) P_{\lambda}\left(x ; q^{2}, t^{2}\right)
$$

then, by induction on $n$, it is enough to prove that

$$
\Phi\left(x^{\prime} ; q, t\right)=\Phi(x ; q, t) \frac{(-t y ; q)_{\infty}}{(y ; q)_{\infty}} \prod_{i=1}^{n} \frac{\left(t^{2} x_{i} y ; q^{2}\right)_{\infty}}{\left(x_{i} y ; q^{2}\right)_{\infty}} .
$$

We will expand both sides of (3.12) in terms of $P_{\mu}\left(x ; q^{2}, t^{2}\right) y^{r}$. After comparing coefficients this results in an identity for Pieri coefficients, given in Proposition 3.3 below.

Lemma 3.1. The right-hand side of (3.12) may be expanded as

$$
\sum_{r=0}^{\infty} \sum_{\substack{\mu, \nu \\ \mu-\nu \in \mathcal{H}}} \frac{(-t ; q)_{r-|\mu-\nu|}}{(q ; q)_{r-|\mu-\nu|}} b_{\nu}^{-}(q, t) \phi_{\mu / \nu}\left(q^{2}, t^{2}\right) P_{\mu}\left(x ; q^{2}, t^{2}\right) y^{r} .
$$


Proof. By (3.11), the $q$-binomial theorem (3.2), and the generating function (3.8) for the $g_{r}$, the right of $(3.12)$ is equal to

$$
\sum_{\nu} \sum_{k, r=0}^{\infty} \frac{(-t ; q)_{k}}{(q ; q)_{k}} b_{\nu}^{-}(q, t) P_{\nu}\left(x ; q^{2}, t^{2}\right) g_{r}\left(x ; q^{2}, t^{2}\right) y^{k+r}
$$

Recalling the Pieri rule (3.9a) this can be further rewritten as

$$
\begin{aligned}
\sum_{k, r=0}^{\infty} & \sum_{\substack{\mu, \nu \\
\mu-\nu \in \mathcal{H}_{r}}} \frac{(-t ; q)_{k}}{(q ; q)_{k}} b_{\nu}^{-}(q, t) \phi_{\mu / \nu}\left(q^{2}, t^{2}\right) P_{\mu}\left(x ; q^{2}, t^{2}\right) y^{k+r} \\
= & \sum_{k=0}^{\infty} \sum_{\substack{\mu, \nu \\
\mu-\nu \in \mathcal{H}}} \frac{(-t ; q)_{k}}{(q ; q)_{k}} b_{\nu}^{-}(q, t) \phi_{\mu / \nu}\left(q^{2}, t^{2}\right) P_{\mu}\left(x ; q^{2}, t^{2}\right) y^{k+|\mu-\nu|} \\
= & \sum_{r=0}^{\infty} \sum_{\substack{\mu, \nu \\
\mu-\nu \in \mathcal{H}}} \frac{(-t ; q)_{r-|\mu-\nu|}}{(q ; q)_{r-|\mu-\nu|}} b_{\nu}^{-}(q, t) \phi_{\mu / \nu}\left(q^{2}, t^{2}\right) P_{\mu}\left(x ; q^{2}, t^{2}\right) y^{r}
\end{aligned}
$$

where the last equality is true since $1 /(q ; q)_{k}=0$ for $k$ a negative integer.

Lemma 3.2. The left-hand side of (3.12) may be expanded as

$$
\sum_{r=0}^{\infty} \sum_{\substack{\lambda, \mu \\ \lambda-\mu \in \mathcal{H}_{r}}} b_{\lambda}^{-}(q, t) \psi_{\lambda / \mu}\left(q^{2} ; t^{2}\right) P_{\mu}\left(x ; q^{2}, t^{2}\right) y^{r}
$$

Proof. Applying [11, page 348, Example 2]

$$
P_{\lambda}\left(x^{\prime} ; q, t\right)=\sum_{\substack{\mu \\ \lambda-\mu \in \mathcal{H}}} \psi_{\lambda / \mu}(q, t) P_{\mu}(x ; q, t) y^{|\lambda-\mu|},
$$

the left of (3.12) is equal to

$$
\begin{aligned}
& \sum_{\substack{\lambda, \mu \\
\lambda-\mu \in \mathcal{H}}} b_{\lambda}^{-}(q, t) \psi_{\lambda / \mu}\left(q^{2}, t^{2}\right) P_{\mu}\left(x ; q^{2}, t^{2}\right) y^{|\lambda-\mu|} \\
& \quad=\sum_{r=0}^{\infty} \sum_{\substack{\lambda, \mu \\
\lambda-\mu \in \mathcal{H}_{r}}} b_{\lambda}^{-}(q, t) \psi_{\lambda / \mu}\left(q^{2}, t^{2}\right) P_{\mu}\left(x ; q^{2}, t^{2}\right) y^{r} .
\end{aligned}
$$

Equating the expansions of Lemmas 3.1 and 3.2 , and extracting coefficients of $P_{\mu}\left(x ; q^{2}, t^{2}\right) y^{r}$ it follows that the proof of Theorem 1.3 boils down to a proof of the following identity for $b_{\lambda}^{-}(q, t)$.

Proposition 3.3. For $\mu$ a partition and $r$ a nonnegative integer,

$$
\sum_{\substack{\nu \\ \mu \in \mathcal{H}}} \frac{(-t ; q)_{r-|\mu-\nu|}}{(q ; q)_{r-|\mu-\nu|}} b_{\nu}^{-}(q, t) \phi_{\mu / \nu}\left(q^{2}, t^{2}\right)=\sum_{\substack{\lambda \\ \lambda-\mu \in \mathcal{H}_{r}}} b_{\lambda}^{-}(q, t) \psi_{\lambda / \mu}\left(q^{2}, t^{2}\right) .
$$


Notationally it turns out to be slightly simpler to prove this in a form involving the Pieri coefficient $\psi_{\lambda / \mu}^{\prime}(q, t)$ given by

$$
P_{\mu}(x ; q, t) e_{r}(x ; q, t)=\sum_{\substack{\lambda \\ \lambda \in \mathcal{V}_{r}}} \psi_{\lambda / \mu}^{\prime}(q, t) P_{\lambda}(x ; q, t),
$$

where $e_{r}=P_{\left(1^{r}\right)}$ is the $r$ th elementary symmetric function

$$
e_{r}(x)=\sum_{i_{1}<i_{2}<\cdots<i_{r}} x_{i_{1}} x_{i_{2}} \cdots x_{i_{r}}
$$

Hence we replace all partitions in (3.13) by their conjugates and use [11, page 341]

$$
\phi_{\mu^{\prime} / \nu^{\prime}}(q, t)=\frac{b_{\nu}(t, q)}{b_{\mu}(t, q)} \psi_{\mu / \nu}^{\prime}(t, q) \quad \text { and } \quad \psi_{\lambda^{\prime} / \mu^{\prime}}(q, t)=\psi_{\lambda / \mu}^{\prime}(t, q)
$$

as well as (3.5). Finally dividing both sides by $b_{\mu^{\prime}}^{-}(q, t)$ it follows that (3.13) can be rewritten as follows.

Proposition 3.3'. For $\mu$ a partition and $r$ a nonnegative integer,

$$
\sum_{\substack{\nu \\ \mu-\nu \in \mathcal{V}}} \frac{(-t ; q)_{r-|\mu-\nu|}}{(q ; q)_{r-|\mu-\nu|}} \frac{b_{\nu}^{-}(t, q)}{b_{\mu}^{-}(t, q)} \psi_{\mu / \nu}^{\prime}\left(t^{2}, q^{2}\right)=\sum_{\substack{\lambda \\ \lambda-\mu \in \mathcal{V}_{r}}} \frac{b_{\lambda^{\prime}}^{-}(q, t)}{b_{\mu^{\prime}}^{-}(q, t)} \psi_{\lambda / \mu}^{\prime}\left(t^{2}, q^{2}\right) .
$$

Crucial in our proof below is an explicit formula for $\psi_{\lambda / \mu}^{\prime}$ due to Macdonald [11, Section VI.6, Equation (6.13)]

$$
\psi_{\lambda / \mu}^{\prime}(q, t)=\prod_{\substack{i<j \\ \lambda_{i}=\mu_{i} \\ \lambda_{j}=\mu_{j}+1}} \frac{\left(1-q^{\mu_{i}-\mu_{j}} t^{j-i-1}\right)\left(1-q^{\lambda_{i}-\lambda_{j}} t^{j-i+1}\right)}{\left(1-q^{\mu_{i}-\mu_{j}} t^{j-i}\right)\left(1-q^{\lambda_{i}-\lambda_{j}} t^{j-i}\right)} .
$$

Proof of Proposition $3.3^{\prime}$. Let us denote the left- and right-hand sides of the identity of Proposition $3.3^{\prime}$ by LHS and RHS. Then

$$
\text { LHS }=\sum_{s=0}^{r} \frac{(-t ; q)_{s}}{(q ; q)_{s}} \sum_{\substack{\nu \\ \mu-\nu \in \mathcal{V}_{r-s}}} \frac{b_{\nu}^{-}(t, q)}{b_{\mu}^{-}(t, q)} \psi_{\mu / \nu}^{\prime}\left(t^{2}, q^{2}\right) .
$$

Let $n$ denote the length of the partition $\mu$, i.e., $\mu=\left(\mu_{1}, \ldots, \mu_{n}\right)$ with $\mu_{n} \geq 1$. We can then replace the sum over $\nu$ by a sum over $k$-subsets $I$ of $[n]$ such that $i \in I$ iff $\mu_{i}-\nu_{i}=1$. In other words, $I$ encodes the parts of $\nu$ that differ from those of $\mu$. Using this notation as well as (3.6) and (3.14) we find

$$
\begin{aligned}
\frac{b_{\nu}^{-}(t, q)}{b_{\mu}^{-}(t, q)}= & \prod_{i \in I} \frac{1-q^{n-i} t^{\mu_{i}}}{1+q^{n-i+1} t^{\mu_{i}-1}} \prod_{\substack{i \in I \\
j \notin I \\
i<j}} \frac{\left(1+q^{j-i+1} t^{\mu_{i}-\mu_{j}-1}\right)\left(1-q^{j-i-1} t^{\mu_{i}-\mu_{j}}\right)}{\left(1-q^{j-i} t^{\mu_{i}-\mu_{j}}\right)\left(1+q^{j-i} t^{\mu_{i}-\mu_{j}-1}\right)} \\
& \times \prod_{\substack{i \in I \\
j \notin I \\
i>j}} \frac{\left(1+q^{j-i} t^{\mu_{i}-\mu_{j}}\right)\left(1-q^{j-i} t^{\mu_{i}-\mu_{j}-1}\right)}{\left(1-q^{j-i+1} t^{\mu_{i}-\mu_{j}-1}\right)\left(1+q^{j-i-1} t^{\mu_{i}-\mu_{j}}\right)}
\end{aligned}
$$


and

$$
\psi_{\mu / \nu}^{\prime}(q, t)=\prod_{\substack{i \in I \\ j \notin I \\ i>j}} \frac{\left(1-q^{\mu_{i}-\mu_{j}-1} t^{j-i+1}\right)\left(1-q^{\mu_{i}-\mu_{j}} t^{j-i-1}\right)}{\left(1-q^{\mu_{i}-\mu_{j}} t^{j-i}\right)\left(1-q^{\mu_{i}-\mu_{j}-1} t^{j-i}\right)} .
$$

Hence

$$
\begin{aligned}
& \sum_{\substack{\nu \\
\mu-\nu \in \mathcal{V}_{k}}} \frac{b_{\nu}^{-}(t, q)}{b_{\mu}^{-}(t, q)} \psi_{\mu / \nu}^{\prime}\left(t^{2}, q^{2}\right) \\
& \quad=\sum_{\substack{I \subseteq[n] \\
|I|=k}} \prod_{i \in I} \frac{1-q^{n-i} t^{\mu_{i}}}{1+q^{n-i+1} t^{\mu_{i}-1}} \prod_{\substack{i \in I \\
j \notin I}} \frac{\left(1+q^{j-i+1} t^{\mu_{i}-\mu_{j}-1}\right)\left(1-q^{j-i-1} t^{\mu_{i}-\mu_{j}}\right)}{\left(1-q^{j-i} t^{\mu_{i}-\mu_{j}}\right)\left(1+q^{j-i} t^{\mu_{i}-\mu_{j}-1}\right)},
\end{aligned}
$$

resulting in

$$
\text { LHS }=\sum_{s=0}^{r} \frac{(-t ; q)_{s}}{(q ; q)_{s}} \sum_{\substack{I \subseteq[n] \\|I|=r-s}} \prod_{i \in I} \frac{1-q^{n-i} t^{\mu_{i}}}{1+q^{n-i+1} t^{\mu_{i}-1}} \prod_{\substack{i \in I \\ j \notin I}} \frac{\left(1+q^{j-i+1} t^{\mu_{i}-\mu_{j}-1}\right)\left(1-q^{j-i-1} t^{\mu_{i}-\mu_{j}}\right)}{\left(1-q^{j-i} t^{\mu_{i}-\mu_{j}}\right)\left(1+q^{j-i} t^{\mu_{i}-\mu_{j}-1}\right)} .
$$

We next turn to the right-hand side, which is a sum over partitions $\lambda$ such that $\lambda-\mu \in \mathcal{V}_{r}$. Recall that $\mu$ has exactly $n$ parts. The maximum number of parts of $\lambda$ is thus $n+r$ (when $\left.\lambda=\mu \cup\left(1^{r}\right)\right)$ and the minimum number of parts of $\lambda$ is $n$. Hence we can write

$$
\mathrm{RHS}=\sum_{s=0}^{r} \sum_{\substack{\lambda-\mu \in \mathcal{V}_{r} \\(\lambda)=n+s}} \frac{b_{\lambda^{\prime}}^{-}(q, t)}{b_{\mu^{\prime}}^{-}(q, t)} \psi_{\lambda / \mu}^{\prime}\left(t^{2}, q^{2}\right)
$$

Again we let $I \subseteq[n]$ (with $|I|=r-s$ ) be the set of indices of those parts of $\mu$ to which a square is added to form $\lambda ; i \in I$ iff $\lambda_{i}-\mu_{i}=1$ for $i \in[n]$. For example if $\mu=(3,2,2,1)$ and $\lambda=(4,3,2,2,1,1)$ then $n=4, r=5, s=2$ and $I=\{1,2,4\}$.

From (3.7), after a tedious calculation, we obtain

$$
\begin{aligned}
\frac{b_{\lambda^{\prime}}^{-}(q, t)}{b_{\mu^{\prime}}^{-}(q, t)}= & \frac{(-t ; q)_{s}}{(q ; q)_{s}} \prod_{i \in I} \frac{1+q^{n-i+s} t^{\mu_{i}+1}}{1-q^{n-i+s+1} t^{\mu_{i}}} \prod_{j \notin I} \frac{\left(1+q^{n-j+s} t^{\mu_{j}}\right)\left(1-q^{n-j+1} t^{\mu_{j}-1}\right)}{\left(1-q^{n-j+s+1} t^{\mu_{j}-1}\right)\left(1+q^{n-j} t^{\mu_{j}}\right)} \\
& \times \prod_{\substack{i \in I \\
j \notin I \\
i<j}} \frac{\left(1+q^{j-i-1} t^{\mu_{i}-\mu_{j}+1}\right)\left(1-q^{j-i+1} t^{\mu_{i}-\mu_{j}}\right)}{\left(1-q^{j-i} t^{\mu_{i}-\mu_{j}}\right)\left(1+q^{j-i} t^{\mu_{i}-\mu_{j}+1}\right)} \\
& \times \prod_{\substack{i \in I \\
j \notin I \\
i>j}} \frac{\left(1-q^{j-i} t^{\mu_{i}-\mu_{j}+1}\right)\left(1+q^{j-i} t^{\mu_{i}-\mu_{j}}\right)}{\left(1-q^{j-1} t^{\mu_{i}-\mu_{j}+1}\right)\left(1+q^{j-i+1} t^{\mu_{i}-\mu_{j}}\right)} .
\end{aligned}
$$

Furthermore, from (3.14),

$$
\begin{aligned}
\psi_{\lambda / \mu}^{\prime}(q, t)= & \prod_{j \notin I} \frac{\left(1-q^{\mu_{j}-1} t^{n-j+s+1}\right)\left(1-q^{\mu_{j}} t^{n-j}\right)}{\left(1-q^{\mu_{j}} t^{n-j+s}\right)\left(1-q^{\mu_{j}-1} t^{n-j+1}\right)} \\
& \times \prod_{\substack{i \in I \\
j \notin I \\
i>j}} \frac{\left(1-q^{\mu_{i}-\mu_{j}} t^{j-i+1}\right)\left(1-q^{\mu_{i}-\mu_{j}+1} t^{j-i-1}\right)}{\left(1-q^{\mu_{i}-\mu_{j}+1} t^{j-i}\right)\left(1-q^{\mu_{i}-\mu_{j}} t^{j-i}\right)} .
\end{aligned}
$$


Putting these results together yields

$$
\begin{aligned}
\mathrm{RHS}= & \sum_{s=0}^{r} \frac{(-t ; q)_{s}}{(q ; q)_{s}} \sum_{\substack{I \subseteq[n] \\
|I|=r-s}} \prod_{i \in I} \frac{1+q^{n-i+s} t^{\mu_{i}+1}}{1-q^{n-i+s+1} t^{\mu_{i}}} \prod_{j \notin I} \frac{\left(1+q^{n-j+s+1} t^{\mu_{j}-1}\right)\left(1-q^{n-j} t^{\mu_{j}}\right)}{\left(1-q^{n-j+s} t^{\mu_{j}}\right)\left(1+q^{n-j+1} t^{\mu_{j}-1}\right)} \\
& \times \prod_{\substack{i \in I \\
j \notin I}} \frac{\left(1+q^{j-i-1} t^{\mu_{i}-\mu_{j}+1}\right)\left(1-q^{j-i+1} t^{\mu_{i}-\mu_{j}}\right)}{\left(1-q^{j-i} t^{\mu_{i}-\mu_{j}}\right)\left(1+q^{j-i} t^{\mu_{i}-\mu_{j}+1}\right)} .
\end{aligned}
$$

Finally equating LHS and RHS we obtain

$$
\begin{aligned}
\sum_{s=0}^{r} \frac{(-t ; q)_{s}}{(q ; q)_{s}} \sum_{\substack{I \subseteq[n] \\
|I|=r-s}} \prod_{i \in I} \frac{1-q^{n-i} t^{\mu_{i}}}{1+q^{n-i+1} t^{\mu_{i}-1}} \prod_{\substack{i \in I \\
j \notin I}} \frac{\left(1+q^{j-i+1} t^{\mu_{i}-\mu_{j}-1}\right)\left(1-q^{j-i-1} t^{\mu_{i}-\mu_{j}}\right)}{\left(1-q^{j-i} t^{\mu_{i}-\mu_{j}}\right)\left(1+q^{j-i} t^{\mu_{i}-\mu_{j}-1}\right)} \\
=\sum_{s=0}^{r} \frac{(-t ; q)_{s}}{(q ; q)_{s}} \sum_{\substack{I \subseteq[n] \\
|I|=-s}} \prod_{i \in I} \frac{1+q^{n-i+s} t^{\mu_{i}+1}}{1-q^{n-i+s+1} t^{\mu_{i}}} \prod_{j \notin I} \frac{\left(1+q^{n-j+s+1} t^{\mu_{j}-1}\right)\left(1-q^{n-j} t^{\mu_{j}}\right)}{\left(1-q^{n-j+s} t^{\mu_{j}}\right)\left(1+q^{n-j+1} t^{\mu_{j}-1}\right)} \\
\quad \times \prod_{\substack{i \in I \\
j \notin I}} \frac{\left(1+q^{j-i-1} t^{\mu_{i}-\mu_{j}+1}\right)\left(1-q^{j-i+1} t^{\mu_{i}-\mu_{j}}\right)}{\left(1-q^{j-i} t^{\mu_{i}-\mu_{j}}\right)\left(1+q^{j-i} t^{\mu_{i}-\mu_{j}+1}\right)} .
\end{aligned}
$$

This is a limiting case of Theorem 1.1. To see this, take the theorem with $p=0$ (recall that $\theta(z ; 0)=(1-z))$ and replace the summation index $r$ by $s$. Then carry out the simultaneous substitutions

$$
\left(t, v, w, x_{i}\right) \mapsto\left(-t,-\epsilon q^{-r} t, q^{-r}, q^{i-n} t^{-\mu_{i}} / \epsilon\right)
$$

(for all $i \in[n]$ ) and take the $\epsilon \rightarrow 0$ limit. Finally replacing $s \mapsto r-s$ and multiplying both sides by $(-t ; q)_{r} /(q ; q)_{r}$ yields $(3.15)$.

\section{Elliptic hypergeometric series}

\subsection{A new multivariable transformation formula}

To turn the theta-function identity of Theorem 1.1 into an identity for elliptic hypergeometric series we apply the well-known procedure of multiple principal specialisation, see e.g., [5, 6, 13, 16].

For $n, m$ integers, let

$$
[n]_{m}:=\{m+1, m+2, \ldots, m+n\},
$$

so that $[n]_{0}=[n]$. In Theorem 1.1 replace

$$
\begin{gathered}
\left(x_{1}, x_{2}, \ldots, x_{n}\right) \mapsto\left(t_{1}, t_{1} q, \ldots, t_{1} q^{m_{1}-1}, t_{2}, t_{2} q, \ldots, t_{2} q^{m_{2}-1}, \ldots,\right. \\
\left.\ldots, t_{N}, t_{N} q, \ldots, t_{N} q^{m_{N}-1}\right),
\end{gathered}
$$

where $m_{1}+\cdots+m_{N}=n$. (In the notation of $\lambda$-rings [9] we are making the substitution $\left.x \mapsto \sum_{i=1}^{N} t_{i}\left(1-q^{m_{i}}\right) /(1-q).\right)$

Since $\theta(1)=0$ it follows that

$$
\prod_{\substack{i \in I \\ j \notin I}} \theta\left(q x_{i} / x_{j}\right) \quad\left(\operatorname{resp} . \prod_{\substack{i \in I \\ j \notin I}} \theta\left(q x_{j} / x_{i}\right)\right)
$$


vanishes unless $I$ is of the form

$$
I=\bigcup_{i=1}^{N}\left[k_{i}\right]_{m_{1}+\cdots+m_{i}-k_{i}} \quad\left(\text { resp. } \quad I=\bigcup_{i=1}^{N}\left[k_{i}\right]_{m_{1}+\cdots+m_{i-1}}\right),
$$

where $k_{1}, \ldots, k_{N}$ are integers such that $0 \leq k_{i} \leq m_{i}$ for each $i$. Since $|I|=r$ we must of course further impose that $|k|:=k_{1}+\cdots+k_{N}=r$.

The rest is essentially a straightforward calculation, and we only sketch the details pertaining to the right-hand side of Theorem 1.1:

$$
\begin{aligned}
\operatorname{RHS} \stackrel{(4.1)}{\longrightarrow} & \sum_{r=0}^{|m|} \frac{(v, w)_{r}}{(q v / t, q w / t)_{r}}\left(\frac{q}{t}\right)^{(N+1) r} \prod_{i=1}^{N} \frac{\left(v t_{i} / t w, v t_{i} q^{r-1}\right)_{m_{i}}}{\left(v t_{i} / q w, v t_{i} q^{r} / t\right)_{m_{i}}} \\
& \times \sum_{\substack{k_{1}, \ldots, k_{N} \\
|k|=r \\
0 \leq k_{i} \leq m_{i}}} \prod_{i=1}^{N} \frac{\left(t_{i} / q, v t_{i} q^{r} / t^{2}, v t_{i} / q w, v t_{i} q^{r} / t\right)_{k_{i}}}{\left(t_{i} / t, v t_{i} q^{r-1} / t, v t_{i} / t w, v t_{i} q^{r-1}\right)_{k_{i}}} \\
& \times \prod_{i, j=1}^{N} \frac{\left(q^{-m_{j}} t_{i} / t_{j}, t t_{i} / t_{j}\right)_{k_{i}}}{\left(q^{1-m_{j}} t_{i} / t t_{j}, q t_{i} / t_{j}\right)_{k_{i}}} \frac{\left(t t_{i} / t_{j}\right)_{k_{i}-k_{j}}}{\left(t t_{i} / t_{j}\right)_{k_{i}-k_{j}}},
\end{aligned}
$$

where we have replaced $n$ by $|m|:=m_{1}+\cdots+m_{N}$. Using

$$
\sum_{\substack{r=0 \\ k_{1}, \ldots, k_{N} \\|k|=r \\ 0 \leq k_{i} \leq m_{i}}} f_{r, k_{1}, \ldots, k_{N}}=\sum_{\substack{k_{1}, \ldots, k_{N} \\ 0 \leq k_{i} \leq m_{i}}} f_{|k|, k_{1}, \ldots, k_{N}}
$$

and making the substitutions

$$
\left(t, t_{i}, v, w\right) \mapsto\left(b, a b q t_{i} / c, c, d\right),
$$

we obtain

$$
V_{m}(a ; b, c, d ; t) \prod_{i=1}^{N} \frac{\left(a q t_{i} / d, a b t_{i}\right)_{m_{i}}}{\left(a q t_{i}, a b t_{i} / d\right)_{m_{i}}}
$$

where, for $t=\left(t_{1}, \ldots, t_{N}\right)$ and $m=\left(m_{1}, \ldots, m_{N}\right)$,

$$
\begin{aligned}
V_{m}(a ; b, c, d ; t):= & \sum_{k_{1}=0}^{m_{1}} \cdots \sum_{k_{N}=0}^{m_{N}} \prod_{i=1}^{N}\left(\frac{\theta\left(a t_{i} q^{k_{i}+|k|}\right)}{\theta\left(a t_{i}\right)} \frac{\left(a b t_{i} / c, a b t_{i} / d\right)_{k_{i}}}{\left(a q t_{i} / c, a q t_{i} / d\right)_{k_{i}}}\right. \\
& \left.\times \frac{\left(a t_{i}, a b q^{m_{i}} t_{i}\right)_{|k|}}{\left(a q t_{i} / b, a q^{m_{i}+1} t_{i}\right)_{|k|}} \frac{\left(a q t_{i} / b\right)_{k_{i}+|k|}}{\left(a b t_{i}\right)_{k_{i}+|k|}}\right) \\
& \times \frac{(c, d)_{|k|}}{(c q / b, d q / b)_{|k|}}\left(\frac{q}{b}\right)^{(N+1)|k|} \prod_{i, j=1}^{N} \frac{\left(q^{-m_{j}} t_{i} / t_{j}, b t_{i} / t_{j}\right)_{k_{i}}}{\left(q^{1-m_{j}} t_{i} / b t_{j}, q t_{i} / t_{j}\right)_{k_{i}}} \frac{\left(b t_{i} / t_{j}\right)_{k_{i}-k_{j}}}{\left(b t_{j}\right)_{k_{i}-k_{j}}} .
\end{aligned}
$$

We note that a particularly succinct way to express this elliptic hypergeometric series follows by introducing $k_{N+1}:=-k_{1}-\cdots-k_{N}$. Then

$$
V_{m}\left(1 / t_{N+1} ; b, c, d ; t\right):=\sum_{k_{1}=0}^{m_{1}} \cdots \sum_{k_{N}=0}^{m_{N}} \prod_{i=1}^{N+1} \frac{\left(b t_{i} / c t_{N+1}, b t_{i} / d t_{N+1}\right)_{k_{i}}}{\left(q t_{i} / c t_{N+1}, q t_{i} / d t_{N+1}\right)_{k_{i}}}
$$




$$
\times \prod_{i=1}^{N+1} \prod_{j=1}^{N} \frac{\left(q^{-m_{j}} t_{i} / t_{j}, b t_{i} / t_{j}\right)_{k_{i}}}{\left(q^{1-m_{j}} t_{i} / b t_{j}, q t_{i} / t_{j}\right)_{k_{i}}} \prod_{i, j=1}^{N+1} \frac{\left(q t_{i} / t_{j}\right)_{k_{i}-k_{j}}}{\left(b t_{i} / t_{j}\right)_{k_{i}-k_{j}}} .
$$

A similar calculation may be carried out for the left-hand side of the theorem and we find exactly the same multiple basic hypergeometric series, but with $a$ replaced by $\hat{a}:=c d / a b$ and $t_{i}$ replaced by $s_{i}:=q^{-m_{i}} / t_{i}$ for all $i$. As a result we can claim the following transformation formula for $\mathrm{A}_{N-1}$ elliptic hypergeometric series.

Theorem 4.1. Let $\hat{a}=c d / a b$ and $s_{i} t_{i}=q^{-m_{i}}$ for all $i \in[N]$. Then

$$
V_{m}(a ; b, c, d ; t)=V_{m}(\hat{a} ; b, c, d ; s) \prod_{i=1}^{N} \frac{\left(a q t_{i}, \hat{a} q s_{i} / c, \hat{a} q s_{i} / d, a q t_{i} / c d\right)_{m_{i}}}{\left(\hat{a} q s_{i}, a q t_{i} / c, a q t_{i} / d, \hat{a} q s_{i} / c d\right)_{m_{i}}} .
$$

For $N=1$, after rescaling $(a, \hat{a}) \mapsto\left(a / t_{1}, \hat{a} / s_{1}\right)$ and replacing $m_{1} \mapsto n$, this gives

$$
\begin{aligned}
\sum_{k=0}^{n} \frac{\theta\left(a q^{2 k}\right)}{\theta(a)} \frac{\left(a, b, c, d, a b / c, a b / d, a b q^{n}, q^{-n}\right)_{k}}{\left(q, a q / b, a q / c, a q / d, c q / b, d q / b, q^{1-n} / b, a q^{n+1}\right)_{k}} \frac{(a q / b)_{2 k}}{(a b)_{2 k}}\left(\frac{q}{b}\right)^{2 k} \\
=\frac{(a q, \hat{a} q / c, \hat{a} q / d, a q / c d)_{n}}{(\hat{a} q, a q / c, a q / d, \hat{a} q / c d)_{n}} \\
\quad \times \sum_{k=0}^{n} \frac{\theta\left(\hat{a} q^{2 k}\right)}{\theta(\hat{a})} \frac{\left(\hat{a}, b, c, d, \hat{a} b / c, \hat{a} b / d, \hat{a} b q^{n}, q^{-n}\right)_{k}}{\left(q, \hat{a} q / b, \hat{a} q / c, \hat{a} q / d, c q / b, d q / b, q^{1-n} / b, \hat{a} q^{n+1}\right)_{k}} \frac{(\hat{a} q / b)_{2 k}}{(\hat{a} b)_{2 k}}\left(\frac{q}{b}\right)^{2 k},
\end{aligned}
$$

where $\hat{a}=q^{-n} c d / a b$. Curiously, even this one-dimensional case, which may also be written as a transformation between ${ }_{20} V_{19}$ elliptic hypergeometric series (or for $p=0$ as a transformation between ${ }_{14} W_{13}$ basic hypergeometric series), is new. To the best of our knowledge it is the first-ever example of a transformation that does not yield a summation upon specialisation of some of its parameters. We award AU $\$ \mathbf{2 5}$ for a proof of (4.2) based on known identities for one-variable elliptic hypergeometric series, and $\mathrm{AU} \$ 10$ for a proof of the $p=0$ case using identities for basic hypergeometric series. We do remark that (4.2) may be viewed as a somewhat strange generalisation of Jackson's ${ }_{6} \phi_{5}$ summation [3, Equation (II.21)]. Indeed, after taking $p=0$ the $b \rightarrow 0$ limit can be taken. Close inspection reveals that on the right the summand vanishes unless $k=0$, resulting in Jackson's sum

$$
{ }_{6} W_{5}\left(a ; c, d, q^{-n} ; q, a q^{n+1} / c d\right)=\frac{(a q, a q / c d ; q)_{n}}{(a q / c, a q / d ; q)_{n}} .
$$

The same in fact applies for general $N$, and setting $p=0$ and then taking the $b \rightarrow 0$ limit in Theorem 4.1 leads to the following $\mathrm{A}_{N-1}$ extension of Jackson's ${ }_{6} \phi_{5}$ summation.

For $t=\left(t_{1}, \ldots, t_{N}\right)$ let

$$
\Delta(t)=\prod_{1 \leq i<j \leq N}\left(t_{i}-t_{j}\right)
$$

be the Vandermonde product, and set

$$
\frac{\Delta\left(t q^{k}\right)}{\Delta(t)}=\prod_{1 \leq i<j \leq N}\left(\frac{t_{i} q^{k_{i}}-t_{j} q^{k_{j}}}{t_{i}-t_{j}}\right) .
$$

Corollary 4.2. For $m_{1}, \ldots, m_{N}$ nonnegative integers, $|m|:=m_{1}+\cdots+m_{N}$ and $e_{2}(k)=\sum_{i<j} k_{i} k_{j}$ we have

$$
\sum_{k_{1}, \ldots, k_{N} \geq 0} \frac{\Delta\left(t q^{k}\right)}{\Delta(t)} \prod_{i=1}^{N}\left(\frac{1-a t_{i} q^{k_{i}+|k|}}{1-a t_{i}}\right) \frac{(c, d ; q)_{|k|}}{\prod_{i=1}^{N}\left(a q t_{i} / c, a q t_{i} / d ; q\right)_{k_{i}}} q^{-e_{2}(k)}
$$




$$
\begin{aligned}
& \times \prod_{i=1}^{N}\left(\frac{\left(a t_{i} ; q\right)_{|k|}}{\prod_{j=1}^{N}\left(q t_{i} / t_{j} ; q\right)_{k_{i}}} \frac{\prod_{j=1}^{N}\left(q^{-m_{j}} t_{i} / t_{j} ; q\right)_{k_{i}}}{\left(a q^{m_{i}+1} t_{i} ; q\right)_{|k|}}\left(\frac{a t_{i} q^{|m|+1}}{c d}\right)^{k_{i}}\right) \\
= & \prod_{i=1}^{N} \frac{\left(a q t_{i}, a q t_{i} / c d ; q\right)_{m_{i}}}{\left(a q t_{i} / c, a q t_{i} / d ; q\right)_{m_{i}}} .
\end{aligned}
$$

This same result also follows by taking the $d \rightarrow \infty$ limit in the $U(n)$ (or $A_{N-1}$ ) Jackson sum [12, Theorem 6.14], or, alternatively, by taking the $b \rightarrow \infty$ limit in the $D_{N}$ Jackson sum $[1$, Theorem A.12].

By a standard analytic argument, see e.g., [14], the sum over the $N$-dimensional hyperrectangle in Theorem 4.1 may be transformed into a sum over the $N$-simplex $k_{1}, \ldots, k_{N} \geq 0$, $k_{1}+\cdots+k_{N} \leq n$.

Corollary 4.3. For $\hat{a}=c d q^{-n} / a b$

$$
W_{n}(a ; b, c, d ; s, t)=W_{n}(\hat{a} ; b, c, d ; t, s) \prod_{i=1}^{N} \frac{\left(a q t_{i}, \hat{a} q / d t_{i}, \hat{a} q s_{i} / c, a q / c d s_{i}\right)_{n}}{\left(\hat{a} q s_{i}, a q / d s_{i}, a q t_{i} / c, \hat{a} q / c d t_{i}\right)_{n}},
$$

where

$$
\begin{aligned}
W_{n}(a ; b, c, d ; s, t):= & \sum_{\substack{k_{1}, \ldots, k_{N} \geq 0 \\
|k| \leq n}} \prod_{i=1}^{N}\left(\frac{\theta\left(a t_{i} q^{k_{i}+|k|}\right)}{\theta\left(a t_{i}\right)} \frac{\left(a b t_{i} / c, a b q^{n} t_{i}\right)_{k_{i}}}{\left(a q t_{i} / c, a q^{n+1} t_{i}\right)_{k_{i}}}\right. \\
& \left.\times \frac{\left(a t_{i}, a b / d s_{i}\right)_{|k|}}{\left(a q t_{i} / b, a q / d s_{i}\right)_{|k|}} \frac{\left(a q t_{i} / b\right)_{k_{i}+|k|}}{\left(a b t_{i}\right)_{k_{i}+|k|}}\right) \\
& \times \frac{\left(c, q^{-n}\right)_{|k|}}{\left(c q / b, q^{1-n} / b\right)_{|k|}}\left(\frac{q}{b}\right)^{(N+1)|k|} \prod_{i, j=1}^{N} \frac{\left(d t_{i} s_{j}, b t_{i} / t_{j}\right)_{k_{i}}}{\left(d q t_{i} s_{j} / b, q t_{i} / t_{j}\right)_{k_{i}}} \frac{\left(q t_{i} / t_{j}\right)_{k_{i}-k_{j}}}{\left(b t_{i} / t_{j}\right)_{k_{i}-k_{j}}} .
\end{aligned}
$$

From the $p=0$ case of Theorem 4.1 we may also deduce the following double multi-sum identity.

Corollary 4.4. For $m_{1}, \ldots, m_{N}$ nonnegative integers and $|m|=m_{1}+\cdots+m_{N}$ we have

$$
\begin{aligned}
\sum_{\substack{l_{i}, k_{i} \geq 0 \\
l_{i}+k_{i} \leq m_{i} \\
i \in[N]}} \prod_{i=1}^{N}\left(\frac{\left(a t_{i} q^{|m|} ; q\right)_{l_{i}+k_{i}}}{\left(a b t_{i} q^{|m|} ; q\right)_{l_{i}+k_{i}}} \frac{\left(a q t_{i} / c d ; q\right)_{l_{i}+k_{i}-|k|}}{\left(a b t_{i} / c d ; q\right)_{l_{i}+k_{i}-|k|}} \frac{\left(a b t_{i} / c, a b t_{i} / d ; q\right)_{l_{i}}}{\left(a q t_{i} / c, a q t_{i} / d ; q\right)_{l_{i}}}\right. \\
\times \frac{\left(a b t_{i}-c d q^{|k|-l_{i}}\right)}{\left(a b t_{i}-c d q^{|k|-l_{i}-k_{i}}\right)} \frac{\left(c d / a t_{i} ; q\right)_{|k|}}{\left(c d q / a b t_{i} ; q\right)_{|k|}} \frac{\left(c d q^{1-l_{i}+|k|-k_{i}} / a b^{2} t_{i} ; q\right)_{k_{i}}}{\left(c d q^{\left.-l_{i}+|k|-k_{i} / a t_{i} ; q\right)_{k_{i}}}\right)} \\
\times \prod_{i, j=1}^{N} \frac{\left(q^{-m_{j}} t_{i} / t_{j} ; q\right)_{l_{i}+k_{i}}}{\left(q^{-m_{j}} b t_{i} / t_{j} ; q\right)_{l_{i}+k_{i}}} \frac{\left(b t_{i} / t_{j} ; q\right)_{l_{i}}}{\left(q t_{i} / t_{j} ; q\right)_{l_{i}}} \frac{\left(q^{l_{i}-l_{j}} b t_{i} / t_{j} ; q\right)_{k_{i}}}{\left(q^{1+l_{i}-l_{j}} t_{i} / t_{j} ; q\right)_{k_{i}}} \\
\times \\
\times \frac{(c, d ; q)_{|k|}}{(c q / b, d q / b ; q)_{|k|}} q^{|l|+N|k|} b^{(1-N)|k|} \frac{1}{\Delta(t)} \\
\times \operatorname{det}_{1 \leq i, j \leq N}\left[\left(t_{i} q^{l_{i}+k_{i}}\right)^{N-j}\left(1-b^{N-j+1} \frac{\left(1-a t_{i} q^{l_{i}+k_{i}+|m|}\right)}{\left(1-a b t_{i} q^{l_{i}+k_{i}+|m|}\right)} \prod_{r=1}^{N} \frac{\left(t_{i} q^{l_{i}+k_{i}}-t_{r} q^{m_{r}}\right)}{\left(b t_{i} q^{l_{i}+k_{i}}-t_{r} q^{\left.m_{r}\right)}\right)}\right)\right]
\end{aligned}
$$




$$
\begin{aligned}
= & q^{\sum_{i=1}^{N}(i+1) m_{i}} b^{-2|m|} \prod_{i, j=1}^{N} \frac{\left(b t_{i} / t_{j} ; q\right)_{m_{i}}}{\left(q t_{i} / b t_{j} ; q\right)_{m_{i}}} \prod_{1 \leq i<j<N} \frac{\left(q t_{i} / b t_{j} ; q\right)_{m_{i}-m_{j}}}{\left(b t_{i} / t_{j} ; q\right)_{m_{i}-m_{j}}} \\
& \times \frac{(c, d ; q)_{|m|}}{(c q / b, d q / b ; q)_{|m|}} \prod_{i=1}^{N} \frac{\left(a b t_{i} / c, a b t_{i} / d ; q\right)_{m_{i}}}{\left(a q t_{i} / c, a q t_{i} / d ; q\right)_{m_{i}}} \frac{\left(a b t_{i} ; q\right)_{|m|}}{\left(a q t_{i} / b ; q\right)_{|m|}} \frac{\left(a q t_{i} / b ; q\right)_{m_{i}+|m|}}{\left(a b t_{i} ; q\right)_{m_{i}+|m|}} .
\end{aligned}
$$

Proof. We define

$$
\begin{aligned}
f_{k}(a ; b, c, d ; t)= & q^{N|k|} b^{-(N+1)|k|} \prod_{i=1}^{N} \frac{\left(a b t_{i} / c, a b t_{i} / d ; q\right)_{k_{i}}}{\left(a q t_{i} / c, a q t_{i} / d ; q\right)_{k_{i}}} \frac{\left(a t_{i} ; q\right)_{|k|}}{\left(a q t_{i} / b ; q\right)_{|k|}} \frac{\left(a q t_{i} / b ; q\right)_{k_{i}+|k|}}{\left(a b t_{i} ; q\right)_{k_{i}+|k|}} \\
& \times \frac{(c, d ; q)_{|k|}}{(c q / b, d q / b ; q)_{|k|}} \prod_{i, j=1}^{N} \frac{\left(b t_{i} / t_{j} ; q\right)_{k_{i}}}{\left(q t_{i} / t_{j} ; q\right)_{k_{i}}} \frac{\left(q t_{i} / t_{j} ; q\right)_{k_{i}-k_{j}}}{\left(b t_{i} / t_{j} ; q\right)_{k_{i}-k_{j}}}
\end{aligned}
$$

and

$$
\begin{aligned}
M_{m k}(a ; b, c, d ; t)= & q^{|k|} \prod_{i=1}^{N} \frac{\left(1-a t_{i} q^{k_{i}+|k|}\right)}{\left(1-a t_{i}\right)} \frac{\left(a b q^{m_{i}} t_{i} ; q\right)_{|k|}}{\left(a q^{1+m_{i}} t_{i} ; q\right)_{|k|}} \frac{\left(a q t_{i} / c, a q t_{i} / d ; q\right)_{m_{i}}}{\left(a q t_{i}, a q t_{i} / c d ; q\right)_{m_{i}}} \\
& \times \prod_{i, j=1}^{N} \frac{\left(q^{-m_{j}} t_{i} / t_{j} ; q\right)_{k_{i}}}{\left(q^{1-m_{j}} t_{i} / b t_{j} ; q\right)_{k_{i}}} .
\end{aligned}
$$

Then the $p=0$ case of Theorem 4.1 may be put as

$$
\sum_{\substack{0 \leq k_{i} \leq m_{i} \\ i \in[N]}} M_{m k}(a ; b, c, d ; t) f_{k}(a ; b, c, d ; t)=\sum_{\substack{0 \leq k_{i} \leq m_{i} \\ i \in[N]}} M_{m k}(\hat{a} ; b, c, d ; s) f_{k}(\hat{a} ; b, c, d ; s),
$$

where $\hat{a}=c d / a b$ and $s_{i} t_{i}=q^{-m_{i}}$ for all $i \in[N]$. According to [10, Theorem 2.3] (modulo the simultaneous substitutions $b \mapsto t_{1} \cdots t_{N} / a, a_{i}\left(y_{i}\right) \mapsto b t_{i} q^{y_{i}}, c_{i}\left(y_{i}\right) \mapsto t_{i} q^{y_{i}}$ for $\left.i \in[N]\right)$ the inverse of the infinite-dimensional lower-triangular matrix $M_{m k}$ is given by

$$
\begin{aligned}
M_{m k}^{-1}(a & ; b, c, d ; t)=\frac{\Delta\left(t q^{m}\right)}{\Delta(t)^{2}} \prod_{i=1}^{N} \frac{\left(a t_{i} ; q\right)_{k_{i}+|m|}}{\left(a b t_{i} ; q\right)_{k_{i}+|m|}} \frac{\left(a b t_{i}, a q t_{i} / c d ; q\right)_{k_{i}}}{\left(a q t_{i} / c, a q t_{i} / d ; q\right)_{k_{i}}} \\
& \times q^{|k|-|m|} \prod_{i, j=1}^{N} \frac{\left(q^{-m_{j}} t_{i} / t_{j}, b t_{i} / t_{j} ; q\right)_{k_{i}}}{\left(q t_{i} / t_{j}, q^{-m_{j}} b t_{i} / t_{j} ; q\right)_{k_{i}}} \frac{\left(q t_{i} / b t_{j} ; q\right)_{m_{i}}}{\left(q t_{i} / t_{j} ; q\right)_{m_{i}}} \\
& \times \operatorname{det}_{1 \leq i, j \leq N}\left[\left(t_{i} q^{k_{i}}\right)^{N-j}\left(1-b^{N-j+1} \frac{\left(1-a t_{i} q^{k_{i}+|m|}\right)}{\left(1-a b t_{i} q^{k_{i}+|m|}\right)} \prod_{r=1}^{N} \frac{\left(t_{i} q^{k_{i}}-t_{r} q^{m_{r}}\right)}{\left(b t_{i} q^{k_{i}}-t_{r} q^{m_{r}}\right)}\right)\right] .
\end{aligned}
$$

Hence the $p=0$ case of Theorem 4.1 is equivalent to

$$
\sum_{\substack{0 \leq k_{i} \leq m_{i} \\ i \in[N]}} \sum_{\substack{k_{i} \leq l_{i} \leq m_{i} \\ i \in[N]}} M_{m l}^{-1}(a ; b, c, d ; t) M_{l k}(\hat{a} ; b, c, d ; s) f_{k}(\hat{a} ; b, c, d ; s)=f_{m}(a ; b, c, d ; t),
$$

where $\hat{a}=c d / a b$ and $s_{i} t_{i}=q^{-l_{i}}$ for all $i \in[N]$. Using

$$
\sum_{\substack{0 \leq k_{i} \leq m_{i} \\ k_{i} \leq l_{i} \leq m_{i} \\ i \in[N]}} c_{m, l, k}=\sum_{\substack{l_{i}, k_{i} \geq 0 \\ l_{i}+k_{i} \leq m_{i} \\ i \in[N]}} c_{m, l+k, k}
$$

and the explicit forms for $f_{k}, M_{m k}$ and $M_{m k}^{-1}$ this gives (after some elementary manipulations) the result as stated in the corollary. 
We remark that for $N=1$ the identity in Corollary 4.4 (in which case the determinant appearing in the summand of the double sum factorises) admits the following elliptic extension:

$$
\begin{aligned}
\sum_{l, k \geq 0} & \frac{\theta\left(a b q^{2 l+2 k}\right)}{\theta(a b)} \frac{\left(a q^{m}, q^{-m}\right)_{l+k}}{\left(b q^{1-m}, a b q^{m+1}\right)_{l+k}} \frac{(b, a b / c, a b / d, a q / c d)_{l}}{(q, a q / c, a q / d, a b / c d)_{l}} q^{l} \\
& \times \frac{\theta\left(c d q^{k-l} / a b\right)}{\theta\left(c d q^{-l} / a b\right)} \frac{\left(c d q^{1-l} / a b^{2}\right)_{k}}{\left(c d q^{-l} / a\right)_{k}} \frac{(b, c, d, c d / a)_{k}}{(q, c q / b, d q / b, c d q / a b)_{k}} q^{k} \\
= & \frac{(a q / b)_{2 m}}{(a b)_{2 m}} \frac{(a b q, b, c, d, a b / c, a b / d)_{m}}{(1 / b, a q / b, a q / c, a q / d, c q / b, d q / b)_{m}}\left(\frac{q}{b^{2}}\right)^{m} .
\end{aligned}
$$

This can proved by inverting the transformation in (4.2) using the elliptic matrix inversion of [22, Equation (3.5); $r=1]$. Since the explicit form of the elliptic extension of the multivariate matrix inversion of [10, Theorem 2.3] is not (yet) available, we were unable to extend our basic $(p=0)$ double multi-sum identity in Corollary 4.4 to the elliptic case.

\subsection{Relation to the $q, t$-Littlewood-Richardson coefficients}

The $p=0$ instance of the transformation in Theorem 4.1 is closely related to an identity that arises by exploiting some basic symmetries of the $q, t$-Littlewood-Richardson (LR) coefficients. The latter are defined by [11, Section VI.7]

$$
P_{\mu}(x ; q, t) P_{\nu}(x ; q, t)=\sum_{\lambda} f_{\mu \nu}^{\lambda}(q, t) P_{\lambda}(x ; q, t) .
$$

Iteration of (4.3) yields

$$
P_{\mu}(x ; q, t) P_{\nu}(x ; q, t) P_{\rho}(x ; q, t)=\sum_{\lambda, \tau} f_{\mu \nu}^{\lambda}(q, t) f_{\lambda \rho}^{\tau}(q, t) P_{\tau}(x ; q, t) .
$$

Since the left-hand side is symmetric under interchange of $\nu$ and $\rho$, we also have

$$
P_{\mu}(x ; q, t) P_{\nu}(x ; q, t) P_{\rho}(x ; q, t)=\sum_{\lambda, \tau} f_{\mu \rho}^{\lambda}(q, t) f_{\lambda \nu}^{\tau}(q, t) P_{\tau}(x ; q, t) .
$$

By equating coefficients of $P_{\tau}(x ; q, t)$ we immediately get the general identity

$$
\sum_{\lambda} f_{\mu \nu}^{\lambda}(q, t) f_{\lambda \rho}^{\tau}(q, t)=\sum_{\lambda} f_{\mu \rho}^{\lambda}(q, t) f_{\lambda \nu}^{\tau}(q, t)
$$

We now assume that $\nu$ and $\rho$ are partitions containing a single part only. Then the four $q, t$-LR coefficients in the above transformation all become Pieri coefficients, and hence completely factorise. Specifically we set $\mu=\left(\mu_{1}, \ldots, \mu_{N}\right), \nu=(r)$ and $\rho=(s)$. Using (3.9a) the transformation (4.4) then simplifies to

$$
\sum_{\substack{\lambda \\ \tau-\lambda \in \mathcal{H}_{s} \\ \lambda-\mu \in \mathcal{H}_{r}}} \phi_{\tau / \lambda}(q, t) \phi_{\lambda / \mu}(q, t)=\sum_{\substack{\lambda \\ \tau-\lambda \in \mathcal{H}_{r} \\ \lambda-\mu \in \mathcal{H}_{s}}} \phi_{\tau / \lambda}(q, t) \phi_{\lambda / \mu}(q, t) .
$$

If we finally apply Macdonald's formula [11, Section VI.6, Example 2(a)]

$$
\phi_{\lambda / \mu}(q, t)=\prod_{1 \leq i<j \leq l(\lambda)} \frac{f\left(q^{\lambda_{i}-\lambda_{j}} t^{j-i}\right) f\left(q^{\mu_{i}-\mu_{j+1}} t^{j-i}\right)}{f\left(q^{\lambda_{i}-\mu_{j}} t^{j-i}\right) f\left(q^{\mu_{i}-\lambda_{j+1}} t^{j-i}\right)},
$$


where $f(u):=(t u ; q)_{\infty} /(q u ; q)_{\infty}$, we obtain a transformation for multiple basic hypergeometric series. To make this explicit we note that since $l(\mu) \leq N$ we have $l(\lambda) \leq N+1$ and $l(\tau) \leq N+2$. We also have $|\tau|-|\mu|=s+r$ so that $\tau_{N+2}$ may be eliminated by $\tau_{N+2}=|\mu|+r+s-\left(\tau_{1}+\right.$ $\left.\cdots+\tau_{N+1}\right)$. Finally we note that since $|\lambda|=|\mu|+r$ on the left and $|\lambda|=|\mu|+s$ on the right, we may in a similar manner eliminate the summation index $\lambda_{N+1}$ on both sides. If we then shift the remaining summation indices $\lambda_{1}, \ldots, \lambda_{N}$ by $\lambda_{i} \mapsto \lambda_{i}+\mu_{i}$ (for $i \in[N]$ ) and carry out some simplifications we arrive at the $p=0$ case of Theorem 4.1 subject to the simultaneous substitutions

$$
\left(a, b, c, d, t_{i}, m_{i}\right) \mapsto\left(q^{-r}, t, q^{\tau_{N+1}-r} t, q^{|\mu|+s-\left(\tau_{1}+\cdots+\tau_{N+1}\right)}, q^{\mu_{i}} t^{N+1-i}, \tau_{i}-\mu_{i}\right)
$$

for $i \in[N]$.

We further remark that the identity in Corollary 4.4 is related to a recursion formula for the $q, t$-Littlewood-Richardson coefficients given in [21]. More precisely, [21, Theorem 2.1] describes an explicit recursion in $n$ for the $q, t$-LR coefficient $f_{\mu^{\prime} \nu^{\prime}}^{\lambda^{\prime}}(t, q)$ where $\lambda, \mu$, and $\nu$ are three partitions with $l(\mu) \leq m, l(\nu) \leq n+1$, and $|\mu|+|\nu|=|\lambda|$. Choosing $m=1$ this coefficient reduces to a Pieri coefficient and hence factorises. As a result one obtains an identity equivalent to Corollary 4.4. This should come as no surprise since [21, Theorem 2.1] is derived in exactly the same way as Corollary 4.4 above; by the use of multidimensional inverse relations.

\section{Acknowledgements}

We thank the anonymous referees for helpful comments. The work reported in this paper is supported by the Australian Research Council and by FWF Austrian Science Fund grants P17563-N13 and S9607.

\section{References}

[1] Bhatnagar G., Schlosser M.J., $C_{n}$ and $D_{n}$ very-well-poised ${ }_{10} \phi_{9}$ transformations, Constr. Approx. 14 (1998), $531-567$.

[2] Frenkel I.B., Turaev V.G., Elliptic solutions of the Yang-Baxter equation and modular hypergeometric functions, The Arnold-Gelfand Mathematical Seminars, Birkhäuser Boston, Boston, MA, 1997, 171-204.

[3] Gasper G., Rahman M., Basic hypergeometric series, 2nd ed., Encyclopedia of Mathematics and Its Applications, Vol. 96, Cambridge University Press, Cambridge, 2004.

[4] Gustafson R.A., Multilateral summation theorems for ordinary and basic hypergeometric series in $U(n)$, SIAM J. Math. Anal. 18 (1987), 1576-1596.

[5] Kajihara Y., Euler transformation formula for multiple basic hypergeometric series of type $A$ and some applications, Adv. Math. 187 (2004), 53-97.

[6] Kajihara Y., Noumi M., Multiple elliptic hypergeometric series. An approach from the Cauchy determinant, Indag. Math. (N.S.) 14 (2003), 395-421, math.CA/0306219.

[7] Kawanaka N., On subfield symmetric spaces over a finite field, Osaka J. Math. 28 (1991), 759-791.

[8] Kawanaka N., A q-series identity involving Schur functions and related topics, Osaka J. Math. 36 (1999), $157-176$.

[9] Lascoux A., Symmetric functions and combinatorial operators on polynomials, CBMS Regional Conference Series in Mathematics, Vol. 99, American Mathematical Society, Providence, RI, 2003.

[10] Lassalle M., Schlosser M.J., Inversion of the Pieri formula for Macdonald polynomials, Adv. Math. 202 (2006), 289-325, math.CO/0402127.

[11] Macdonald I.G., Symmetric functions and Hall polynomials, 2nd ed., Oxford University Press, New York, 1995.

[12] Milne S.C., Multiple $q$-series and $U(n)$ generalizations of Ramanujan's ${ }_{1} \psi_{1}$ sum, in Ramanujan Revisited (Urbana-Champaign, Ill., 1987), Academic Press, Boston, MA, 1988, 473-524. 
[13] Rosengren H., A proof of a multivariable elliptic summation formula conjectured by Warnaar, in $q$-Series with Applications to Combinatorics, Number Theory, and Physics (Urbana, IL, 2000), Contemp. Math., Vol. 291, Amer. Math. Soc., Providence, RI, 2001, 193-202, math.CA/0101073.

[14] Rosengren H., Elliptic hypergeometric series on root systems, Adv. Math. 181 (2004), 417-447, math.CA/0207046.

[15] Rosengren H., Schur Q-polynomials, multiple hypergeometric series and enumeration of marked shifted tableaux, J. Combin. Theory Ser. A 115 (2008), 376-406, math.CO/0603086.

[16] Rosengren H., Schlosser M.J., On Warnaar's elliptic matrix inversion and Karlsson-Minton-type elliptic hypergeometric series, J. Comput. Appl. Math. 178 (2005), 377-391, math.CA/0309358.

[17] Ruijsenaars S.N.M., Complete integrability of relativistic Calogero-Moser systems and elliptic function identities, Comm. Math. Phys. 110 (1987), 191-213.

[18] Ruijsenaars S.N.M., Elliptic integrable systems of Calogero-Moser type: some new results on joint eigenfunctions, in Proceedings of the 2004 Kyoto Workshop on Elliptic Integrable Systems, Editors M. Noumi and K. Takasaki, Rokko Lectures in Mathematics, no. 18, Kobe University, 2005, 223-240.

[19] Ruijsenaars S.N.M., Schneider H., A new class of integrable systems and its relation to solitons, Ann. Physics 170 (1986), 370-405.

[20] Schlosser M.J., Multidimensional matrix inversions and $A_{r}$ and $D_{r}$ basic hypergeometric series, Ramanujan J. 1 (1997), 243-274.

[21] Schlosser M.J., Explicit computation of the $q$, $t$-Littlewood-Richardson coefficients, in Jack, Hall-Littlewood and Macdonald Polynomials, Contemp. Math., Vol. 417, Amer. Math. Soc., Providence, RI, 2006, 335-343.

[22] Warnaar S.O., Summation and transformation formulas for elliptic hypergeometric series, Constr. Approx. 18 (2002), 479-502, math.QA/0001006.

[23] Warnaar S.O., Rogers-Szegö polynomials and Hall-Littlewood symmetric functions, J. Algebra 303 (2006), 810-830, arXiv:0708.3110.

[24] Whittaker E.T., Watson G.N., A course of modern analysis, 4th ed., Cambridge University Press, Cambridge, 1927. 